\title{
A 160-MHz MAP OF CAS A
}

\author{
H. HIRABAYASH I
}

Tokyo Astronomical Observatory, Tokyo, Japan

\begin{abstract}
A rotational synthesis map of Cas A was obtained at $160 \mathrm{MHz}$ with a resolution of $2 \times 3^{\prime}$.
I should like to report my preliminary results of a rotational synthesis map of Cas A at $160 \mathrm{MHz}$. The results were obtained using the compound grating interferometer of the Nobeyama Solar Radio Station, Tokyo Astronomical Observatory. The resolution was $2 \times 3^{\prime}$. Thus the frequency and the resolution are much the same as that used by Slee and Dulk on the Culgoora Solar Radioheliograph. The interferometer consists of two independent one-dimensional arrays aligned in the E-W and N-S directions.

The data were obtained as a series of one-dimensional images, and to make the weightings uniform on the two-dimensional $u, v$ plane, the observed images were convolved with the function whose Fourier transform has an amplitude proportional to the absolute value of the spatial frequency vector. They were then integrated on the two-dimensional image plane, taking into consideration the variation of base-line vector (i.e., the orientation and elongation of the images) with respect to the hour angle.

One day was used for the observation, and the observed hour angle ranged over $9 \mathrm{~h}$. At this time only the E-W arm was used. The figure shows the synthesized map of Cas A and the beam on the same scale. The overall appearance of the map is similar to the higher frequency results. Although high resolution maps have been made at higher frequencies, those at such low frequency are important to determine the structure of the supernova remnants in a wider frequency range.

This is the first preliminary result. By adding the N-S arm data one can get much better coverage on the $u, v$ plane. Also, the 'CLEAN' procedure would restore the images of the supernova remnants to reveal more plausible structure in many cases. I hope to extend the observation to some of the other supernova remnants accessible from the Northern Hemisphere.
\end{abstract}

H. Hirabayashi

Tokyo Astronomical Observatory, Mitaka, Tokyo, Japan

(Discussion follows paper by R. M. Duin et al., p. 361.) 


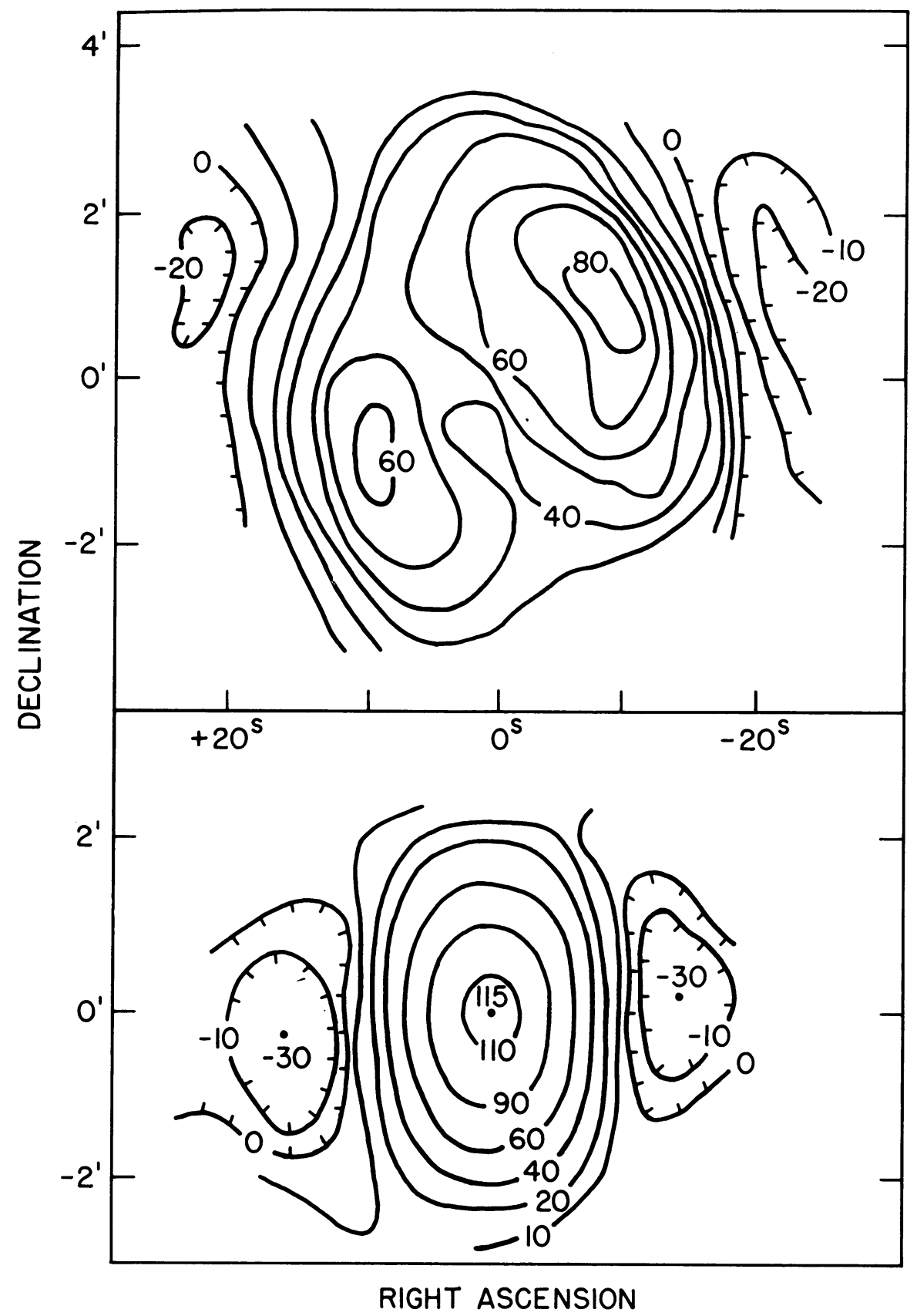

Fig. 1. Brightness contour map of Cas A (above) and the corresponding two-dimensional beam (below). Brightness scales are in arbitrary units. The map was obtained by the E-W arm and is not yet 'CLEANED.' 